\title{
Ten-year survival rate for cantilevered fixed partial dentures
}

\author{
How long do cantilever fixed partial dentures survive and what effects do \\ biological and technical complications have on them?
}

\begin{abstract}
Pjetursson BE, Tan K, Lang NP, Bragger U, Egger M, Zwahlen M. A systematic review of the survival and complication rates of fixed partial dentures (FPDs) after an observation period of at least 5 years. IV. Cantilever or extension FPDs. Clin Oral Implant Res 2004; 15:625-642
\end{abstract}

Data sources PubMed (1966-April 2004) provided the primary data source along with the bibliographies from identified articles and reviews. A manual search of two relevant journals (Clinical Implant Dentistry and Related Research, Clinical Oral Implants Research, International Journal of Oral \& Maxillofacial Implants, International Journal of Periodontics and Restorative Dentistry, International Journal of Prosthodontics, Journal of Clinical Periodontology, Journal of Periodontology and Journal of Prosthetic Dentistry published; published 2001-2003) provided a further source of data.

Study selection Because there were no randomised controlled trials (RCT) that compared fixed partial dentures (FPD) with and without cantilever extensions, other studies were selected if they met the following criteria: were published in the English language; were prospective and retrospective cohort studies; had a mean follow-up of $>5$ years; included patients who were clinically examined at follow-up; and reported details on suprastructures and described at least one-third of reconstructions as FPD.

Data extraction and synthesis Two independent reviewers screened articles for inclusion. Disagreements were resolved by discussion and agreement determined by kappa. Two reviewers extracted data on the survival and success of the reconstructions and on biological and technical complications. Failure and complication rates were calculated by dividing the number of events by the total exposure time.

Results Three prospective and 10 retrospective cohort studies incorporating 700 patients and 816 FPD were included. The mean number of cantilever extensions ranged from 1.1 to 6.0. Meta-analyses gave an estimated survival, after 10 years, of $81.8 \%$ [95\% confidence interval $(\mathrm{Cl}), 78.2-84.9]$ and a proportion success of $63 \%(95 \% \mathrm{Cl}$, 54.7-70.2). Considering biological complications using Poisson model analyses, the estimated rate of FPD lost because of caries after 10 years was $3.1 \%(95 \% \mathrm{Cl}, 1.0-8.8)$ and that for loss of vitality was $32.6 \%(95 \%$ $\mathrm{Cl}, 13.9-64.9)$. The rate of FPD lost because of periodontitis was $1 \%$ (95\% Cl, 0.3-3.0). Cumulative 10-year complication rates were: $2.9 \%$ $(95 \% \mathrm{Cl}, 1.7-5.0)$ for fracture of abutment tooth; $2.4 \%(95 \% \mathrm{Cl}, 0.6$ $9.8)$ for rate of loss as a result of abutment fracture; $16.1 \%\left(95 \% \mathrm{Cl}_{\text {, }}\right.$ 8.8-28.4) for loss of retention; and 5.9\% (95\% Cl, 3.3-10.4) for material complications.

Conclusions Success and survival rates for cantilever FPD are poorer than those for conventional FPD and this is accompanied by frequent biological and technical complications.

Address for correspondence: Bjarni E Pjetursson, Department of Periodontology and Fixed Prosthodontics, University of Berne, Freiburgstrasse 7, CH 3010 Berne, Switzerland. E-mail: bjarni.pjetursson@zmk.unibe.ch

\section{Commentary}

This systematic review is the final one following three other reviews ${ }^{1-3}$ by the same group looking at the survival and complication rates of different designs of FPD. The title of the series is slightly misleading in suggesting that the data reflect a minimum of 5 years of observation, whereas the inclusion criteria for study selection in fact required a mean follow-up time of 5 years or more. The total sample size actually exposed for more than 5 years is thus reduced, although the size of the reduction is not easily discerned from the report.

The objectives, to record the survival of cantilevered FPD and the incidence of biological and technical failures, are clearly stated and reasonable, as are the definitions of the survival, success and complications. The authors point out that cantilevered FPD are likely to be found in less demanding situations with regard to loading stress. One would have to be cautious, therefore, in comparing the data presented here with other FPD or implantsupported situations.

The search strategy was thorough, but limited to studies published in the English language. Since no RCT could be found (nor, in this situation, would they necessarily be advantageous) the authors included cohort studies, both prospective and retrospective. Reports were excluded most often because of mean observation periods of $<5$ years, multiple reports on the same patient cohorts and unclear reporting. All but three of the 13 selected studies had sample sizes of fewer than 35 patients, and most were done in institutional settings. On the basis of the information presented, it appears appropriate to combine the selected studies.

"Survival" of an FPD was defined as simply as its presence in situ at examination. "Success" was an FPD that was unchanged and had required no intervention to the date of the examination. Biological complications included caries, loss of pulp vitality and periodontal disease progression. Technical complications included loss of retention and fractures of abutment teeth, frameworks or veneers. The rates of failure were calculated by dividing the number of events by the total exposure time (which was estimated by four different means as necessary for each study). As noted earlier, the use of data from mean follow-up times of 5 years or more will have the effect of inflating the denominator, where the period of interest is over 5 years, thus underestimating the event rate. Further, it is not possible to determine from the data presented how many patients were actually followed for as much as 10 years, so the extent to which the estimates of 10-year survival are based on regression extrapolations is not clear.

The estimated annual failure and complication rates were presented as the number of events per 100 FPD-years of exposure, (summarised at two failures and 4.6 complications per 100 FPDyears). Although these are hardly intuitively useful figures for informed consent, they may also be misleading, in view of the timedependent nature of the sources of complication and failure.

Of the 506 FPD units reviewed, $72 \%$ were gold/resin constructions, whereas only $28 \%$ were metal/ceramic. Fortunately, the survival rates for the two material types were nearly identical, suggesting little effect of the material on survival. Technical 
material fracture was not reported by material used, however, so the applicability of the fracture failure information to the now-commonly used metal/ ceramic constructions is slightly in doubt.

Overall, the report estimates an encouraging 10-year survival rate for cantilevered FPD of $81.5 \%$, and a $63 \%$ success rate free of all complications. Nevertheless, an estimated $32.6 \%$ of abutment teeth lose vitality and over $9 \%$ become carious at 10 years, a significant finding for informed consent and professional surveillance. Other biological and technical failures were less frequent. Although it is likely that cantilevered designs are chosen for what are thought to be less-stressed environments, these success rates are lower than those for conventional FPD designs. ${ }^{3}$ This too should be considered when treatment planning options are available.

\section{Practice point}

- The estimated 10-year survival rate for cantilevered FPD (81.5\%) and success rate free of all complications (63\%) is encouraging. An estimated $32.6 \%$ of abutment teeth lose vitality and $>9 \%$ become carious at 10 years, a significant finding for informed consent and professional surveillance.

\section{James D Anderson}

Division of Prosthodontics, Faculty of Dentistry, Toronto, Ontario, Canada

1. Lang NP, Pjetursson BE, Tan K, Bragger U, Egger M, Zwahlen M. A systematic review of the survival and complication rates of fixed partial dentures (FPDs) after an observation period of at least 5 years. II, Combined tooth-implant-supported FPDs. Clin Oral Implant Res 2004; 15:643-653.

2. Tan K, Pjetursson BE, Lang NP, Chan ES. A systematic review of the survival and complication rates of fixed partial dentures (FPDs) after an observation period of at least 5 years. III. Conventional FPDs. Clin Oral Implant Res 2004; 15:654-666.

3. Pjetursson BE, Tan K, Lang NP, Bragger U, Egger M, Zwahlen M. A systematic review of the survival and complication rates of fixed partial dentures (FPDs) after an observation period of at least 5 years. I. Implant-supported FPDs. Clin Oral Implant Res 2004; 15:667-676.

Evidence-Based Dentistry (2005) 6, 96-97.

doi:10.1038/sj.ebd.6400365 